\title{
Learning Robust Graph Regularisation for Subspace Clustering
}

\author{
Elyor Kodirov, Tao Xiang \\ \{e.kodirov,t.xiang\}@qmul.ac.uk \\ Zhenyong Fu, Shaogang Gong \\ \{z.fu,s.gong\}@qmul.ac.uk
}

\author{
School of Electronic Engineering and \\ Computer Science, \\ Queen Mary University of London, \\ London E1 4NS, UK
}

\begin{abstract}
Various subspace clustering methods have benefited from introducing a graph regularisation term in their objective functions [2]. In this work, we identify two critical limitations of the graph regularisation term employed in existing subspace clustering models and provide solutions for both of them. First, the squared $l_{2}$-norm used in the existing term is replaced by a $l_{1}$-norm term to make the regularisation term more robust against outlying data samples and noise. Solving $l_{1}$ optimisation problems is notoriously expensive and a new formulation and an efficient algorithm are provided to make our model tractable. Second, instead of assuming that the graph topology and weights are known a priori and fixed during learning, we propose to learn the graph [1] and integrate the graph learning into the proposed $l_{1}$-norm graph regularised optimisation problem. Extensive experiments were conducted on five benchmark datasets.
\end{abstract}

Methodology. To address the aforementioned problems, we propose following objective function:

$$
\begin{aligned}
\min _{\mathbf{D}, \mathbf{Y}, \mathbf{W}} & \frac{1}{2}\|\mathbf{X}-\mathbf{D Y}\|_{F}^{2}+\lambda_{1}\|\mathbf{Y}\|_{1}+\lambda_{2}\left\|\mathbf{Y} \mathbf{A}_{\mathbf{W}}\right\|_{1}+\lambda_{3}\|\mathbf{W}\|_{F}^{2} \\
\text { s.t. } & \left\|\mathbf{d}_{i}\right\|^{2} \leq 1, \mathbf{W}^{\mathbf{T}} \mathbf{1}=1, \mathbf{W} \geq 0 .
\end{aligned}
$$

where $\mathbf{X} \in \mathbb{R}^{r \times N}$ is a data matrix with $N \stackrel{r}{r}$ dimensional data feature vectors as columns, $\mathbf{D} \in \mathbb{R}^{r \times d}$ is a dictionary with $d$ number of atoms, $\mathbf{W}$ is an affinity matrix that captures the topology of the data, $\mathbf{A}_{\mathbf{W}}$ is a matrix that is obtained by applying eigendecomposition on $\mathbf{W}$, and $\mathbf{Y} \in \mathbb{R}^{d \times N}$ is a sparse code matrix. In the following, we give explanation for each term:

(1) $\|\mathbf{X}-\mathbf{D Y}\|_{F}^{2}$ is the reconstruction error term evaluating how well a linear combination of the atoms (columns) of the dictionary $\mathbf{D}$, can approximate the data matrix $\mathbf{X}$.

(2) $\lambda_{1}\|\mathbf{Y}\|_{1}$ is a sparsity regularisation term on $\mathbf{Y}$, with a weighting factor $\lambda_{1}$ to favour a small number of atoms to be used for the reconstruction.

(3) $\lambda_{2}\left\|\mathbf{Y} \mathbf{A}_{\mathbf{W}}\right\|_{1}$ is our proposed robust graph regularisation term. Note that we are using $l_{1}$-norm instead of $l_{2}$-norm weighted by $\lambda_{2}$.
(4) $\lambda_{2}\left\|\mathbf{Y} \mathbf{A}_{\mathbf{W}}\right\|_{1}+\lambda_{3}\|\mathbf{W}\|_{F}^{2}$ is the term with proper constraints $\left(\mathbf{W}^{\mathbf{T}} \mathbf{1}=1\right.$ and $\left.\mathbf{W} \geq 0\right)$ for graph learning weighted by $\lambda_{2}$ and $\lambda_{3}$.

The constraints, $\mathbf{W}^{\mathbf{T}} \mathbf{1}=1$ and $\mathbf{W} \geq 0$, are there to ensure the validity of the learned graph, while the constraint $\left\|\mathbf{d}_{i}\right\|^{2} \leq 1\left(\mathbf{d}_{i}\right.$ is a column of $\mathbf{D}$ with $i=1, \ldots, r$ ) enforces the learned dictionary atoms to be compact.

Remark. Terms (3) and (4) are robust graph regularisation and graph learning terms, while first two terms, (1) and (2) constitute the conventional objective function of dictionary learning.

Optimisation. To solve the objective Eq. (1), we develop an algorithm based on ADMM.

Experiments. Table 1 shows experiments on benchmark datasets which are CMU-PIE (C-PIE for short), COIL, ORL, Yale, and YaleB. CA stands for clustering accuracy $(\%)$.

Table 1: Comparative results on C-PIE, COIL, ORL, Yale, and YaleB. 'G' stands for graph.

\begin{tabular}{r|c||c|c|c|c|c}
\hline \multicolumn{2}{l|}{} & \multicolumn{6}{|c}{ CA (\%) } \\
\hline Methods & G & C-PIE & COIL & ORL & Yale & YaleB \\
\hline$l_{1} \mathrm{G}$ & No & 70.3 & 67.1 & 66.8 & 40.0 & 48.4 \\
SSC & No & 72.1 & 58.9 & 55.5 & 38.7 & 52.6 \\
LRR & No & 71.5 & 45.1 & 66.2 & 46.2 & 65.0 \\
LSR & No & 77.9 & 56.0 & 56.5 & 48.5 & 62.4 \\
CASS & No & 82.6 & 59.1 & 68.3 & 45.6 & 81.9 \\
\hline \hline GSC & Yes & $\mathbf{1 0 0}$ & 80.9 & 61.5 & 43.4 & 74.2 \\
R $l_{1}$ G & Yes & 89.5 & 79.4 & 62.0 & 41.3 & 68.5 \\
SMR & Yes & 85.4 & 65.6 & 57.6 & 45.3 & 73.5 \\
NSLRR & Yes & 85.1 & 61.8 & 55.3 & NA & NA \\
\hline \hline PCAN & Yes & 82.5 & 76.5 & 49.1 & 54.4 & 59.2 \\
LML & Yes & 90.2 & 80.2 & 46.7 & 46.7 & 60.9 \\
SDRAM & Yes & 95.6 & 86.3 & 70.6 & 51.8 & 92.3 \\
\hline \hline Ours & Yes & $\mathbf{1 0 0}$ & $\mathbf{8 8 . 1}$ & $\mathbf{7 6 . 3}$ & $\mathbf{5 9 . 6}$ & $\mathbf{9 5 . 2}$
\end{tabular}

\section{References}

[1] Feiping Nie, Xiaoqian Wang, and Heng Huang. Clustering and projected clustering with adaptive neighbors. In Proceedings of the 20th ACM SIGKDD international conference on Knowledge discovery and data mining, pages 977-986. ACM, 2014.

[2] Miao Zheng, Jiajun Bu, Chun Chen, Can Wang, Lijun Zhang, Guang Qiu, and Deng Cai. Graph regularized sparse coding for image representation. Image Processing, IEEE Transactions on, 20(5):1327-1336, 2011. 